\section{THE TIGIT/CD226/CD155 AXIS AND THE EFFECTS OF COMBINING PD-1/PD-L1 BLOCKADE WITH TIGIT- TARGETING ANTIBODY THERAPY IN SYNGENEIC MURINE GLIOBLASTOMA MODELS}

${ }^{1}$ Prafulla Gokhale, ${ }^{1}$ Bryan lorgulescu*, ${ }^{2}$ Max Klapholz, ${ }^{1}$ Michael Poitras, ${ }^{1}$ Benjamin Eschle, ${ }^{1}$ Gordon Freeman, ${ }^{3}$ Ana Anderson, ${ }^{1}$ David Reardon. ${ }^{1}$ Dana-Farber Cancer Institute, Boston, MA, USA; ${ }^{2}$ University of Pennsylvania, Philadelphia, PA, USA; ${ }^{3}$ Brigham and Women's Hospital, Boston, MA, USA

Background Recently, TIGIT+PD1 blockade was shown to confer additive survival benefits in orthotopic glioblastoma and colon cancer mouse models ${ }^{1}{ }^{2}$-notably, this included experiments $^{3}$ that used the 1G9 TIGIT monoclonal antibody $(\mathrm{mAb})$ that has potentially agonistic effects. ${ }^{1}$ Herein we investigated the TIGIT/CD226/CD155 axis and effects of combining clinically analogous PD-1/PD-L1 mAbs with TIGITtargeting $1 \mathrm{G} 9 \mathrm{mAb}$ in murine glioblastoma models.

Methods The overall survival (OS) associated with TIGIT (non-depleting IG9; 200 $\mu \mathrm{g}$ every 3 days for 4 doses), ${ }^{1}$ PD-1 $(8 \mathrm{H} 3$; initial $500 \mu \mathrm{g}$ followed by $250 \mu \mathrm{g}$ every 3 days for 7 doses), PD-L1 (6A2; initial $500 \mu \mathrm{g}$ followed by $250 \mu \mathrm{g}$ every 3 days for 7 doses), and/or IgG mAbs was assessed in immunocompetent C57BL/6 albino mice intracranially implanted with syngeneic GL261-luc2 or CT2A-luc. ${ }^{4}{ }^{5}$ The roles of T cells and NK cells were examined using depletion with CD8a, CD4, or NK1.1 mAbs. Expression of TIGIT/CD226/CD155 and PD-1/PD-L1/PD-L2 by tumor and tumor-infiltrating immune cells was evaluated using flow cytometry and RTqPCR.

Results In vitro, GL261-luc2 and CT2A-luc tumor cells moderately expressed PD-L1, PD-1, and TIGIT; but strongly expressed TIGIT's inhibitory ligand CD155.(Figure1A-B) Ex vivo, $>83 \%$ of CD8+ and CD4+TILs in GL261-luc2 coexpressed TIGIT+/CD226+: $\geq 2 \mathrm{x}$ the proportions in CT2Aluc.(Figure 2A) CD155 and PD-L1 were highly co-expressed on tumor-infiltrating macrophages: greater in GL261-luc2 than CT2A-luc tumors.(Figure 2B)In GL261-luc2 mice, anti-TIGIT monotherapy displayed minimal OS improvement; whereas anti-PD-1 and anti-PD-L1 monotherapies demonstrated robust OS responses.(Figure 3A) Adding anti-TIGIT to PD-1/PD-L1 blockade demonstrated synergism with anti-PD-1. Anti-TIGIT plus anti-PD-1 displayed nominally improved OS in CT2A-luc compared to anti-PD-1 monotherapy $(p=0.11)$.(Figure $3 \mathrm{~B})$. Given robust T-cell expression of TIGIT and PD-1, we examined how $\mathrm{CD} 4+$ or $\mathrm{CD} 8+$ depletion affected responses in GL261-luc2 mice: depletion completely abrogated anti-PD-1's benefits.(Figure 4) Although CD4/CD8 depletion also reduced anti-TIGIT + anti-PD1's efficacy, the resulting OS matched that of non-depleted anti-PD-1 monotherapy. Additionally, NK cell depletion had no effect on anti-TIGIT+anti-PD1's efficacy.

Conclusions Our results recapitulate published findings regarding the synergistic benefits of combining TIGIT $1 \mathrm{G} 9 \mathrm{mAb}$ with anti-PD-1 using the clinically-relevant $8 \mathrm{H} 3 \mathrm{mAb}$ in syngeneic mouse glioblastoma, and extend those findings to antiTIGIT+anti-PDL1 combinations. TIGIT/CD226 was highly coexpressed by immuno-responsive GL261-luc2's tumor-infiltrating lymphocytes (TILs); wheres CD155/PD-L1 expression predominated in tumor-infiltrating myeloid cells. Depletion of $\mathrm{CD} 8+$ or CD4+ TILs modestly reduced anti-TIGIT+antiPD1's efficacy-suggesting a mechanism that is at least partially independent of $\mathrm{T}$ (and NK) cells. Our preliminary results suggest a complex interplay between TIGIT/CD226/ CD155 and PD-1/PD-L1/PD-L2 axes in tumors and their microenvironmental constituents that warrants further investigation; plus, careful consideration of antibody clones' functionality is necessary for designing immunotherapy combinations.

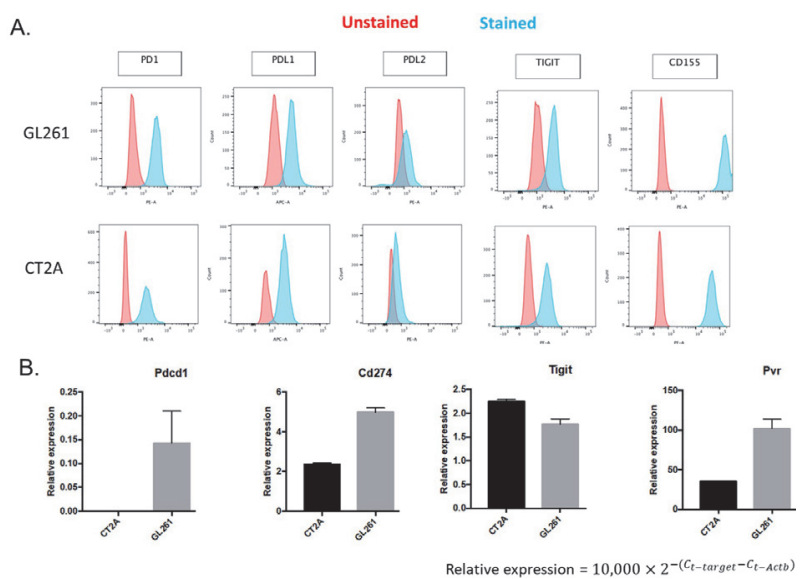

Abstract 256 Figure 1 TIGIT/CD155 axis and PD-1/PD-L1/PD-L2 axis expression in murine glioblastoma model tumor cells. Protein and RNA expression of the TIGIT and PD-1 immune checkpoints - and their ligands CD155 and PDL-1/PD-L2 respectively - on GL261-luc2 and CT2A-luc tumor cells using (A) flow cytometry (blue=samples, red=unstained controls) and (B) RT-qPCR (grey=GL261-luc2, black=CT2A-luc). Pdcd1 encodes PD-1, Cd274 encodes PD-L1, Pvr encodes CD155
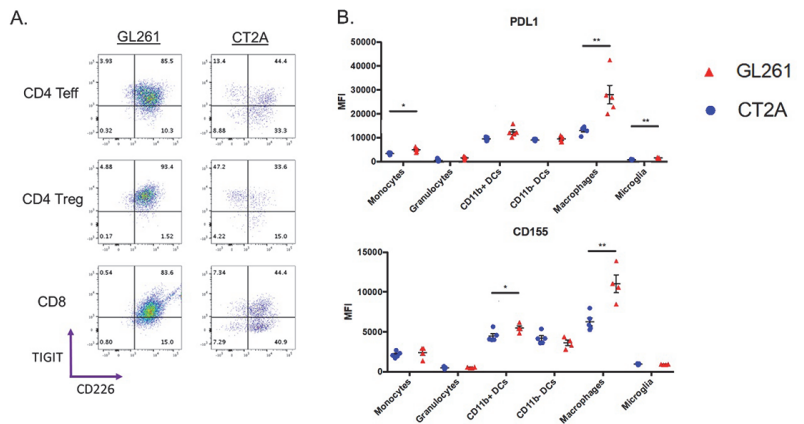

Abstract 256 Figure 2 TIGIT/CD155 axis and PD-1/PD-L1/PD-L2 axis expression in murine glioblastoma model tumor-infiltrating immune cells. (A) Flow cytometry analysis of protein expression for TIGIT (y-axis) and CD226 (x-axis; a competitor of TIGIT) on CD8+, CD4+/FOXP3+ Treg, and CD4+/FOXP3- Teff tumor-infiltrating lymphocytes (TILs) from GL261-luc2 and CT2A-luc tumor-bearing mice. (B) Flow cytometry analysis of protein expression for CD155 (TIGIT's ligand; bottom) and PD-L1 (PD-1's ligand; top) on tumor-infiltrating myeloid populations from GL261-luc2 and CT2A-luc tumor-bearing mice. Mean fluorescent intensity (MFI) was compared between cell lines, *indicates statistical significance. Myeloid populations included CD45+/CD11b+/CD11C+/F4$80+$ macrophages, CD11b+/CD11c+ and CD11b-/CD11c+ dendritic cells (DCs), CD11b+/CD11c-/Ly6C+/Ly6G- monocytes, CD11b+/CD11c-/ Ly6Cmid/Ly6G + granulocytes, and CD45dim/CX3CR1+ microglia. Tumors were dissociated and leukocytes were enriched for using Percoll gradient. $n=5$ mice per group. 


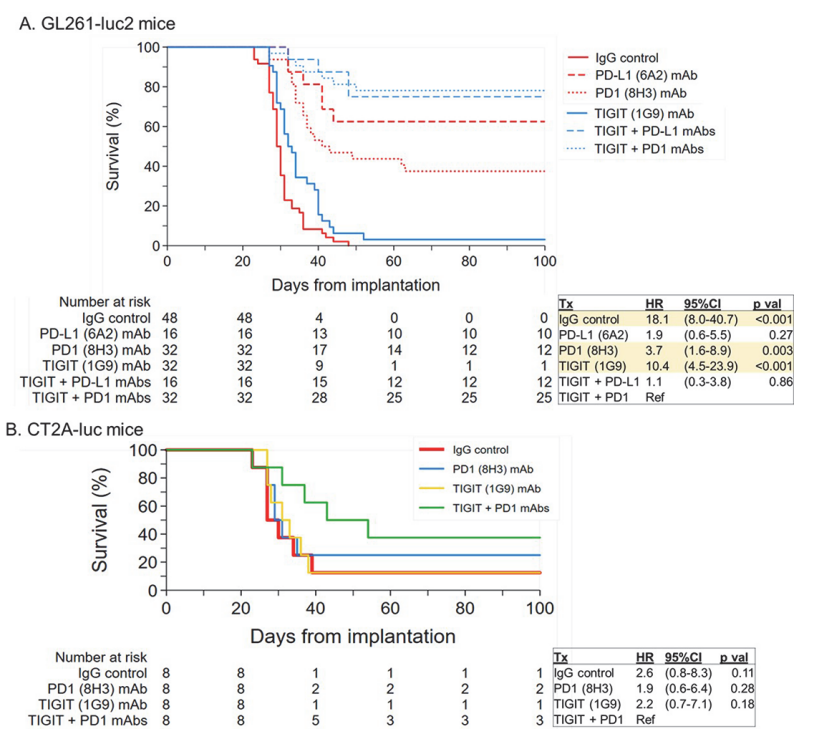

Abstract 256 Figure 3 The survival associated with TIGIT-targeting mAb therapy with/without clinically-analogous PD-1/PD-L1 blockade. (A) Kaplan-Meier estimated overall survival (measured from day of intracranial tumor implantation) of GL261-luc2 mice treated with TIGIT (1G9), PD-1 (8H3), PD-L1 (6A2), TIGIT + PD-1, TIGIT + PD-L1 mAbs, or IgG control. (B) A) Kaplan-Meier estimated overall survival (measured from day of intracranial tumor implantation) of CT2A-luc mice treated with TIGIT (1G9), PD-1 (8H3), TIGIT + PD-1 mAbs, or IgG control. For both experiments, all treatments were started on day 6 following implantation, with the following dosing: anti-TIGIT was given as $200 \mu \mathrm{g}$ every 3days for 4 doses. Both anti-PD-1 and anti-PD-L1 were given as an initial $500 \mu \mathrm{g}$ dose followed by $250 \mu \mathrm{g}$ every 3 days for 7 doses. ${ }^{4}$ The $\mathrm{n}$ per group and number at risk table is included underneath each graph, along with the corresponding Cox regression analysis. Treatment groups with significantly different OS from the combination TIGIT + PD1 combination-treated reference group were highlighted in yellow. $\mathrm{HR}=$ hazard ratio, $\mathrm{Cl}=$ confidence interval.

GL261-luc2 mice
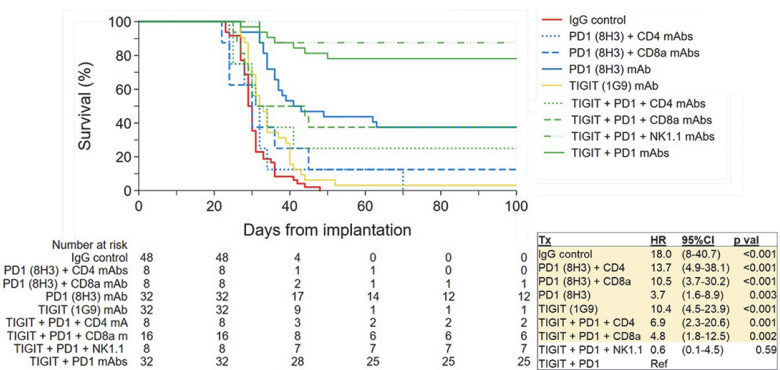

Abstract 256 Figure 4 How depletion of CD8+ T cells, CD4+ T cells, or NK cells affects survival associated with TIGIT-targeting mAb therapy with/without clinically-analogous PD-1 blockade. Kaplan-Meier estimated overall survival (measured from day of intracranial tumor implantation) of GL261-luc2 mice treated with TIGIT (1G9), PD-1 (8H3), TIGIT + PD-1 mAbs, or IgG control; and compared to groups that additional had CD8+, CD4+, or NK1.1+ antibody-based depletion. The treatment and dosing characteristics were the same as Figure 3 . The $n$ per group and number at risk table is included underneath the graph, along with the corresponding Cox regression analysis. Treatment groups with significantly different OS from the combination TIGIT + PD-1 combination-treated reference group were highlighted in yellow. $\mathrm{HR}=$ hazard ratio, $\mathrm{Cl}=$ confidence interval.
Acknowledgements We gratefully acknowledge the support of the The Jennifer Oppenheimer Cancer Research Initiative; The Ben and Catherine Ivy Foundation; Hope It's A Beach Thing; and the Pan Mass Challenge (Erica's Entourage and CRUS11TOUR), and the NCI (P01CA236749; K12CA090354).

\section{REFERENCES}

1. Dixon KO, Schorer M, Nevin J, Etminan Y, Amoozgar Z, Kondo T, Kurtulus S, Kassam N, Sobel RA, Fukumura D, Jain RK, Anderson AC, Kuchroo VK, Joller N. Functional anti-TIGIT antibodies regulate development of autoimmunity and antitumor immunity. J Immunol 2018 April 15;200(8):3000-3007.

2. Hung AL, Maxwell R, Theodros D, Belcaid $Z$, Mathios D, Luksik AS, Kim E, Wu A, Xia Y, Garzon-Muvdi T, Jackson C, Ye X, Tyler B, Selby M, Korman A, Barnhart B, Park SM, Youn Jl, Chowdhury T, Park CK, Brem H, Pardoll DM, Lim M. TIGIT and PD-1 dual checkpoint blockade enhances antitumor immunity and survival in GBM. Oncoimmunology 2018 May 24;7(8):e1466769.

3. Raphael I, Kumar R, McCarl LH, Shoger K, Wang L, Sandlesh P, Sneiderman CT, Allen J, Zhai S, Campagna ML, Foster A, Bruno TC, Agnihotri S, Hu B, Castro BA, Lieberman FS, Broniscer A, Diaz AA, Amankulor NM, Rajasundaram D, Pollack IF, Kohanbash G. TIGIT and PD-1 immune checkpoint pathways are associated with patient outcome and anti-tumor immunity in glioblastoma. Front Immunol 2021 May 7;12:637146.

4. Reardon DA, Gokhale PC, Klein SR, Ligon KL, Rodig SJ, Ramkissoon SH, Jones KL, Conway AS, Liao X, Zhou J, Wen PY, Van Den Abbeele AD, Hodi FS, Qin L, Kohl NE, Sharpe AH, Dranoff G, Freeman GJ. Glioblastoma eradication following immune checkpoint blockade in an orthotopic, immunocompetent model. Cancer Immunol Res 2016 February;4(2):124-35.

5. Iorgulescu JB, Gokhale PC, Speranza MC, Eschle BK, Poitras MJ, Wilkens MK, Soroko KM, Chhoeu C, Knott A, Gao Y, Lim-Fat MJ, Baker GJ, Bonal DM, Nguyen QD, Grant GRL, Ligon KL, Sorger PK, Chiocca EA, Anderson AC, Kirschmeier PT, Sharpe AH, Freeman GJ, Reardon DA. Concurrent dexamethasone limits the clinical benefit of immune checkpoint blockade in glioblastoma. Clin Cancer Res 2021 January 1;27(1):276-287.

http://dx.doi.org/10.1136/jitc-2021-SITC2021.256 